\title{
Der $p 1$ gene sequence polymorphism in house dust mite Dermatophagoides pteronyssinus
}

\author{
ARYANI ADJI ${ }^{1,2, \vartheta}$, NURDJANNAH J. NIODE ${ }^{1,2}$, VENTJE V. MEMAH ${ }^{1}$, JIMMY POSANGI ${ }^{3}$, \\ GRETA J. P. WAHONGAN ${ }^{4}$, TRINA E. TALLEI ${ }^{5}$ \\ ${ }^{1}$ Program of Entomology, Graduate School, Universitas Sam Ratulangi. Jl. Kampus Unsrat, Manado 95115, North Sulawesi, Indonesia \\ ${ }^{2}$ Department of Dermatovenereology, RD Kandou Hospital. Jl. Raya Tanawangko No. 56, Manado 95163, North Sulawesi, Indonesia. \\ Tel.: +62-431-8383058, `email: aryaniadji@gmail.com \\ ${ }^{3}$ Department of Pharmacology, Faculty of Medicine, Universitas Sam Ratulangi. Jl. Kampus Unsrat, Manado 95115, North Sulawesi, Indonesia \\ ${ }^{4}$ Department of Parasitology, RD Kandou Hospital, Jl. Raya Tanawangko No. 56, Manado, 95163, North Sulawesi, Indonesia \\ ${ }^{5}$ Department of Biology, Faculty of Mathematics and Natural Sciences, Universitas Sam Ratulangi. J1. Kampus Unsrat, Manado 95115, North Sulawesi, \\ Indonesia
}

Manuscript received: 29 October 2020. Revision accepted: 10 December 2020.

\begin{abstract}
Adji A, Niode NJ, Memah VV, Posangi J, Wahongan GJP, Tallei TE. 2021. Der p 1 gene sequence polymorphism in house dust mite Dermatophagoides pteronyssinus. Biodiversitas 22: 72-78. Gene polymorphisms in Dermatophagoides pteronyssinus (DP) mite can affect the immune response that plays a role in atopic disease. This study aimed to determine the polymorphisms of Der $\mathrm{p} 1$ gene from DP. House dust containing DP was used as a source of DNA containing the Der $\mathrm{p} 1$ gene. The gene was amplified using specific primers DP Der 1 P 108F and DP Der 1 P 1509R. The amplified fragments were cloned using the Toyobo pTA2 vector and sorted then compared with the Gene Bank database. The result shows that there are 8 polymorphism sites in the Der $p 1$ gene positioned at 190 (GR), 463 (CT), 645 (AR), 751 (CY, CT), 787 (CG), 827 (TA; TW), and 1084 (CG). In the Der p 1 gene, there are 5 silent mutations and 3 nonsynonymous substitutions, resulting in amino acid polymorphisms as follows: Histidine H146 to Tyrosine Y146, Alanine A220 to Valine V220 and amino acid X220, and Tyrosine T232 to Serine S232. Polymorphisms in these amino acids lead to a polarity change, from polar to polar and non-polar to non-polar. These dynamics in polarity does not change the structure or function of the allergen protein. So that they can be used for vaccine design strategies.
\end{abstract}

Keywords: Dermatophagoides pteronyssinus, mite, allergen, Der p 1 gene, polymorphism

\section{INTRODUCTION}

Dermatophagoides pteronyssinus (DP), a widely popular dominant house dust mite (HDM) which generally known as strong inducers of $\mathrm{IgE}$ antibody response (Liao et al. 2015), has been identified to have many IgE-binding components in the allergen extract (Resende et al. 2019). Allergy is mainly caused by Der p 1 and Der p 2 allergens and these allergens are recognized by more than $80 \%$ of atopic individuals (Cui et al. 2018). Exposure to and sensitization by these allergens are precipitating factors in atopic clinical manifestations (Verma et al. 2016). Der p 1 allergen is important allergen in the indoor environment in which humans live as they have caused allergic diseases such as asthma, rhinitis and atopic dermatitis (Chevigne et al. 2018). Der p 1 derived from HMD is a glycoprotein consisting of a sequence of 222 amino acids that a variant of peptidase 1 or known and endopeptidase 1, which is found in various types of mites (López-Rodríguez et al. 2019). This enzyme has Der $p 1$ protein activity with broad endopeptidase specificity (Gough et al. 2014). Gene polymorphism in the major mite allergens can significantly affect the immune response, specifically the immunoglobulin $\mathrm{E}$ binding and $\mathrm{T}$ cell responses (Piboonpocanun et al. 2006). Although, the study on the environmental isolates of DP is still limited, variation of DNA sequence in human leukocyte antigen (HLA) genes promotes susceptibility to a wide range of human diseases (Jia et al. 2013).

Protein identification Technologies advance in such a fast revolution. For instance, one of the most popular approaches in peptides or proteins detection is to search the mass spectra against a sequence database of known proteins derived from the human reference genome or cDNA sequence repositories (Cao et al. 2017). Polymorphisms are variations in DNA sequences that occur when one nucleotide ( $\mathrm{A}, \mathrm{T}, \mathrm{C}$, or $\mathrm{G}$ ) in the genome sequence changes. The term that is often used is single nucleotide polymorphisms (SNP). This is also called a mutation (Lusiastuti et al. 2019). A point mutation is a change in the $\mathrm{N}$ base of DNA or RNA that relatively common but its effects can be reduced by gene recovery mechanisms (Karki et al. 2015). Point mutations can result in changes in the amino acid sequence in proteins and reduce, altered, or lost enzyme function (Lusiastuti et al. 2019). Current technology uses point mutations as markers (called SNPs) to study changes in genes and are associated with phenotypic changes (Cao et al. 2017).

Cross-reactivity of allergens from different mite species can be predicted by amino acid residue analysis (Shafique et al. 2014). Cross-reactivity, along with the specificity of monoclonal antibodies, can be affected by mutations in some IgE binding epitopes. Thus, the diversity of allergens 
between and within species should be considered for the development of a suitable allergen extract (Cao et al. 2017). This just emphasizes that accurate and appropriate identification and characterization of representative variants in any given geographic area are essential and necessary (Karki et al. 2015). The polymorphisms of regional amino acid sequences and the extent of these polymorphisms are still under-researched, and such data simply do not exist in various countries, including the US and Pakistan (Shafique et al. 2014). This study aimed to analyze polymorphism in the Der p 1 gene based on the facts previously mentioned.

\section{MATERIALS AND METHODS}

\section{Collection of dust samples}

Samples were obtained from bed and sofa dust in 30 houses in Manado, Pineleng, Tomohon and Tondano. Sampling was carried out with a specially modified household vacuum cleaner to collect dust.

\section{DNA extraction}

A total of $10 \mathrm{~g}$ of house dust was added to $40 \mathrm{ml}$ of TE buffer then centrifuged at a speed of $12,000 \mathrm{~g}$ for 5 minutes. DNA was extracted from the pellets obtained using the Zymobiomics kit (Zymo Research, D4300) (www.zymoresearch.com) according to available protocols.

\section{Amplification of the Der $p 1$ gene}

Amplification of the Der $\mathrm{p} 1$ gene region was carried out using specific primer pairs of DP Der 1 P $108 \mathrm{~F}$ (CTCTCTAAAATCTAAAATCCATCC) and DP Der $1 \mathrm{P}$ 1509 R (AATTTAATTTTTGTGAATG). The PCR process was carried out using the Toyobo Kod FX Neo kit (KFX201) with the following PCR conditions: initial denaturation at $95{ }^{\circ} \mathrm{C}$ for 3 minutes ( 1 cycle), followed by 35 cycles consisting of denaturation at $98{ }^{\circ} \mathrm{C}$ for 15 seconds, annealing at $52{ }^{\circ} \mathrm{C}$ for 30 seconds, and extension at $68{ }^{\circ} \mathrm{C}$ for 45 seconds, and ended with a hold at $4{ }^{\circ} \mathrm{C}$ until the amplification result was used. The amplification results were extracted from gel electrophoresis and purified using Zymoclean Gel DNA Recovery Kit (Zymo Research Corp)(www.zymoresearch.com).

\section{Der $p 1$ gene cloning}

In order to obtain the intact gene when sequencing, the Der $\mathrm{p} 1$ gene was cloned on Toyobo pTA2 Vector. The ligation process was carried out using the Toyobo Target Clone ligation reaction (Toyobo). The transformation was performed on E. coli DH5 $\alpha$ using Mix \& Go E. coli Transformation Kit (Zymo Research Corp) (www.zymoresearch.com).

\section{PCR colony amplification}

PCR colony amplification was performed using Toyobo Kod FX Neo (KFX-201) with the following reaction conditions: initial denaturation at $95^{\circ} \mathrm{C}$ for 3 minutes $(1$ cycle), followed by 35 cycles consisting of denaturation at $98^{\circ} \mathrm{C}$ for 10 seconds, annealing at $45^{\circ} \mathrm{C}$ for 30 seconds, and extension at $72^{\circ} \mathrm{C}$ for 45 seconds, and ended with a hold at $4^{\circ} \mathrm{C}$.

\section{Der $p 1$ gene sequencing}

Colonies containing inserts were sequenced using specific primary pairs of DP Der 1 P $108 \mathrm{~F} 5^{\prime}$ CTCTCTAAAATCTAAAATCCATCC 3' and DP Der $1 \mathrm{P}$ 1509R 5' AATTTAATTTTTGTGAATG 3'. Specific primers are designed to reinforce nearly the entire gene coding region.

\section{Sequences analysis}

The sequencing results in the form of a chromatogram were edited using Geneious Pro Version 10 (Tallei and Kolondam 2015). The similarities between sequences were searched using BLAST search at https://blast.ncbi.nlm.nih.gov/Blast.cgi (Altshul et al. 1990). The sequences were aligned using the software in the Multalin network http://multalin.toulouse.inra.fr/multalin/ (Corpet 1988). The alignment results were analyzed descriptively by comparing the sequences of Der $\mathrm{p} 1$ genes with other similar sequences obtained from the BLAST search results. The phylogenetic tree was built using the Neighbor-Joining method which is integrated in the MegaX software (Saitou and Nei 1987).

\section{RESULTS AND DISCUSSION}

\section{Der $p 1$ gene polymorphism}

The similarity between the sequencing results was searched using BLAST search. All similar sequences were then aligned, and the results were shown in Figure 1. There are 8 polymorphism sites found in the Der $\mathrm{p} 1$ gene (Figure 1). Amino acid polymorphisms can affect the reactivity of the IgE bonds from allergens. A single amino acid mutation can alter the production of inflammatory cytokines specific $\mathrm{T}$ cells for Der $\mathrm{p} 1$. It is possible that this mutation affects the innate allergenicity of certain variants and contributes to the frequency of $\operatorname{IgE}$ binding by increasing epitope diversity (Jeong et al. 2012; Verma et al. 2016).

Table 1 shows that there are 8 polymorphism sites in the Der p 1 gene positioned at 190 (GR), 463 (CT), 645 (AR), 751 (CY, CT), 787 (CG), 827 (TA; TW), and 1084 (CG). Polymorphism usually occurs due to single nucleotide substitution or also known as single nucleotide polymorphism (SNP). SNP is a variation of DNA sequences that occurs when a single nucleotide or one nucleotide A, T, C or G in the genome is different from the biological species, such as the DP mite (Clancy 2008). Shafique et al. (2014) have observed the polymorphisms of the group 1 allergen gene sequences in the DP populations from the US and Pakistan using species-specific primers and direct PCR. Those gene sequences were observed under the same conditions, which contained two new introns (nt post 87 and 291) in both species, the absence of introns 3 in Der $p 1$ that was a silent mutation at position of nt (aa) 1011 (149), and four nonsynonymous mutations at position of 589 (50), 935 (124), 971 (136), 1268 (215). They concluded that the 
polymorphism's rate in both genes were considerably lower than previous report $(0.10-0.16 \%$ vs $0.31-0.49 \%)$. This indicates potential errors in the polymerase process using RT-PCR, so further evaluation of the matter is substantial to be done very carefully. In addition, these mutations mentioned above have previously been reported from several geographic regions, suggesting that polymorphisms in the Der p 1 gene are panmictic (Thomas 2010).

AA1 ATTTTATATA ATTGCCATTT ATTCTTGTTT CACCTTTGTT ATATATTGTA GAAAGCCTTC AACAAAAGTT ATGCTACCTT CGAAGATGAA GAAGCTGCCC GTAAAAACTT TTTGGAATCA GTAAAATATG AJ542093 ATTTTATATA ATTGCCATTT ATTCTTGTTT CACCTTTGTT ATATATTGTA GAAAGCCTTC AACAAAAGTT ATGCTACCTT CGAAGATGAA GAAGCTGCCC GTAAAAACTT TTTGGAATCA GTAAAATAT KJ542087 ATTTTATATA ATTGCCATTT ATTCTTGTTT CACCTTTGTT ATATATTGTA GAAAGCCTTC AACAAAAGTT ATGCTACCTT CGAAGATGAA GAAGCTGCCC GTAAAAACTT TTTGGAATCA GTAAAATAT KJ542086 ATTTTATATA ATTGCCATTT ATTCTTGTTT CACCTTTGTT ATATATTGTA GAAAGCCTTC AACAAAAGGT ATGCTACCTT CGAAGATGAA GAAGCTGCCC GTAAAAACTT TTTGGAATCA GTAAAATATG KJ542096 ATTTTATATA ATTGCCATTT ATTCTTGTTT CACCTTTGTT ATATATTGTA GAAAGCCTTC AACAAAAGTT ATGCTACCTT CGAAGATGAA GAAGCTGCCC GTAAAAACTT TTTGGAATCA GTAAAAATATG KJ542089 ATTTTATATA ATTGCCATTT ATTCTTGTTT CACCTTTGTT ATATATTGTA GAAAGCCTTC AACAAAAGTT ATGCTACCTT CGAAGATGAA GAAGCTGCCC GTAAAAACTT TTTGGAATCA GTAAAATATC

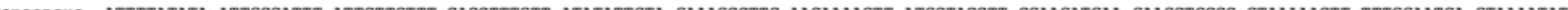
131

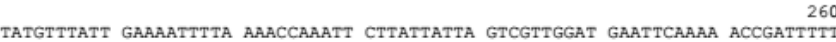

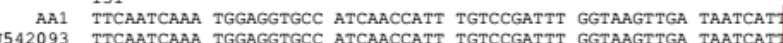

KJ542087 TTCAATCAAA TGGAGGTGCC ATCAACCATT TGTCCGATTT GGTAAGTTGA TAATCAT

KJ542086 TTCAATCAAA TGGAGGTGCC ATCAACCATT TGTCCGATTT GGTAAGTTGA TAATCAT

KJ542096 TTCAATCAAA TGGAGGTGCC ATCAACCATT TGTCCGATTT GGTAAGTTGA TAATCAT TTCAATCAAA TGGAGGTGCC ATCAACCATT TGTCCGATTT GGTAAGTTGA TAATCAT TATGTTTATT GAAAATTTTA AAACCAAATT CTTATTATTA GTCGTTGGAT GAATTCAAAA ACCGATTTT TATGTTTATT GAAAATTTTA AAACCAAATT CTTATTATTA GTCGTTGGAT GAATTCAAAA ACCGATTTT TATGTTTATT GAAAATTTTA AAACCAAATT CTTATTATTA GTCGTTGGAT GAATTCAAAA ACCGATTTTT TATGTTTATT GAAAATTTTA AAACCAAATT CTTATTATTA GTCGTTGGAT GAATTCAAAA ACCGATTTT
TATGTTATT GAAAATTTA AAACCAAATT CTTATTATTA GTCGTTGGAT GAATTCAAAA ACCGATTTT

TGAGTGCA GAAGCTTTTG AACACCTCAA AACTCAATTC GATTTGAATG CTGAAACTAA CGCCTGCAGT ATCAATGGAA ATGCTCCAGC TGAAATCGAT TTGCGACAAA TGCGAACTGT CACTCCCAT GATGAGTGCA GAAGCTTTTG AACACCTCAA AACTCAATTC GATTTGAATG CTGAAACTAA CGCCTGCAGT ATCAATGGAA ATGCTCCAGC TGAAATCGAT TTGCGACAAA TGCGAACTGT CACTCCCAT KJ542087 GATGAGTGCA GAAGCTTTTG AACACCTCAA AACTCAATTC GATTTGAATG CTGAAACTAA CGCCTGCAGT ATCAATGGAA ATGCTCCAGC TGAAATCGAT TTGCGACAAA TGCGAACTGT CACTCCCAT KJ542086 GATGAGTGCA GAAGCTTTTG AACACCTCAA AACTCAATTC GATTTGAATG CTGAAACTAA CGCCTGCAGT ATCAATGGAA ATGCTCCAGC TGAAATCGAT TTGCGACAAA TGCGAACTGT CACTCCCAT GATGAGTGCA GAAGCTTTTG AACACCTCAA AACTCAATTC GATTTGGAATG CTGAAACTAA CGCCTGCAGT ATCAATGGAA ATGCTCCAGC TGAAATCGAT TTGCGACAAA TGCGAACTGT CACTCCCAT GATGAGTGCA GAAGCTTTTG AACACCTCAA AACTCAATTC GATTTGAATG CTGAAACTAA CGCCTGCAGT ATCAATGGAA ATGCTCCAGC TGAAATCGAT TTGCGACAAA TGCGAACTGT CACTCCCAT GATGAGTGCA GAAGCTTTTG AACACCTCAA AACTCAATTC GATTTGAATG CTGAAACTAA CGCCTGCAGT ATCAATGGAA ATGCTCCAGC TGAAATCGAT TTGCGACAAA TGCGAACTGT CACTCCCATT 391

521 TCGAA TCAATCATTG GATCTTGCTG AACAAGAATT AGTCGATTGT GCTTCCCAAC

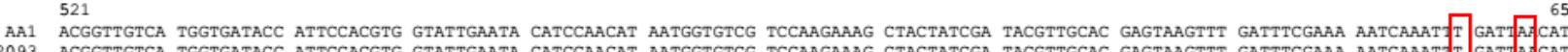
ACGGTTGTCA TGGTGATACC ATTCCACGTG GTATTGAATA CATCCAACAT AATGGTGTCG TCCAAGAAAG CTACTATCGA TACGTTGCAC GAGTAAGTTT GATTTCGAAA AATCAAAT IT GATIA. CAT

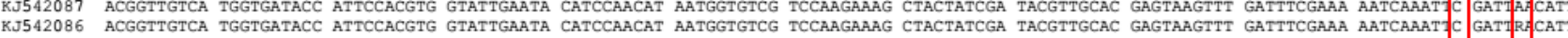
KJ542096 ACGGTTGTCA TGGTGATACC ATTCCACGTG GTATTGAATA CATCCAACAT AATGGTGTCG TCCAAGAAAG CTACTATCGA TACGTTGCAC GAGTAAGTTT GATTTCGAAA AATCAAAT C CATPAACATI KJ542089 ACGGTTGTCA TGGTGATACC ATTCCACGTG GTATTGAATA CATCCAACAT AATGGTGTCG TCCAAGAAAG CTACTATCGA TACGTTGCAC GAGTAAGTTT GATTTCGAAA AATCAAAT IC GATIAACATI

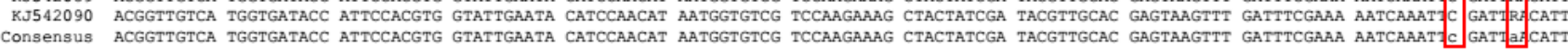
651

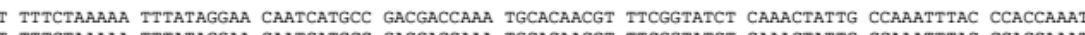
CATCAAATTT TTTCTAAAAA TTTATAGGAA CAATCATGCC GACGACCAAA TGCACAACGT TTCGGTATCT CAAACTATTG CCAAATTTAC CCACCAAATG CATCAAATTT TTTCTAAAAA TTTATAGGAA CAATCATGCC GACGACCAAA TGCACAACGT TTCGGTATCT CAAACTATTGG CCAAATTTAC CCACCAAATT CATCAAATT TTTCTAAAAA TTTATAGGAA CAATCATGCC GACGACCAAA TGCACAACGT TTCGGTATCT CAAACTATTG CCAAATTTAC CCACCAAATG ATCAAATTT TTTCTAAAAA TTTATAGGAA CAATCATGCC GACGACCAAA TGCACAACGT TTCGGTATCT CAAACTATTG CCAAATTTAC CCACCAAATG CATCAAATTT TTTCTAAAAA TTTATAGGAA CAATCATGCC GACGACCAAA TGCACAACGT TTCGGTATCT CAAACTATTG CCAAATTTAC CCACCAAATG
CATCAAATTT TTTCTAAAAA TTTATAGGAA CAATCATGCC GACGACCAAA TGCACAACGT TTCGGTATCT CAAACTATTG CCAAATTTAC CCACCAAATG 781 CCCACAg GC TATTGCCGTC ATTATTGGCA TCAAAGATTT AGACG. TC CGTCATTATG AGTAAGTTTG CCAATCAATT GAATGATGCA ATAAAATTAA TGTTTTATTA TCAATTTTTT AGTGGCCGA 911

Aa1 CAATCATTCA ACGCGATAAT GGTTACCAAC CAAACTATCA CGCTGTCAAC ATTGTTGGTT ACAGTAACGC ACAAGGTGTC GATTATTGGA TCGTACGAAA CAGTTGGGAT ACCAATTGGG GTGATAATG KJ542093 CAATCATTCA ACGCGATAAT GGTTACCAAC CAAACTATCA CGCTGTCAAC ATTGTTGGTT ACAGTAACGC ACAAGGTGTC GATTATTGGA TCGTACGAAA CAGTTGGGAT ACCAATTGGG GTGATAATG KJ542087 CAATCATTCA ACGCGATAAT GGTTACCAAC CAAACTATCA CGCTGTCAAC ATTGTTGGTT ACAGTAACGC ACAAGGTGTC GATTATTGGA TCGTACGAAA CAGTTGGGAT ACCAATTGGG GTGATAATGG KJ542086 CAATCATTCA ACGCGATAAT GGTTACCAAC CAAACTATCA CGCTGTCAAC ATTGTTGGTT ACAGTAACGC ACAAGGTGTC GATTATTGGA TCGTACGAAA CAGTTGGGAT ACCAATTGGG GTGATAATGG KJ542096 CAATCATTCA ACGCGATAAT GGTTACCAAC CAAACTATCA CGCTGTCAAC ATTGTTGGTT ACAGTAACGC ACAAGGTGTC GATTATTGGA TCGTACGAAA CAGTTGGGAT ACCAATTGGG GTGATAATGG KJ542089 CAATCATTCA ACGCGATAAT GGTTACCAAC CAAACTATCA CGCTGTCAAC ATTGTTGGTT ACAGTAACGC ACAAGGTGTC GATTATTGGA TCGTACGAAA CAGTTGGGAT ACCAATTGGG GTGATAATGG Consensus CAATCATTCA ACGCGATAAT GGTTACCAAC CAAACTATCA CGCTGTCAAC ATTGTTGGTT ACAGTAACGC ACAAGGTGTC GATTATTGGA TCGTACGAAA CAGTTGGGAT ACCAATTGGG GTGATAATGG

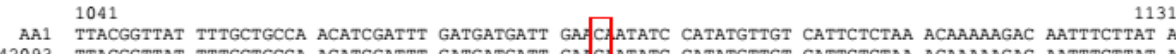
KJ542093 TTACGGTTAT TTTGCTGCCA ACATCGATTT GATGATGATT GA:CAATATC CATATGTTGT CATTCTCTAA ACAAAAAGAC AATTTCTTAT A KJ542087 TTACGGTTAT TTTGCTGCCA ACATCGATTT GATGATGATT GA:G:ATATC CATATGTTGT CATTCTCTAA ACAAAAAGAC AATTTCTTAT A KJ542086 TTACGGTTAT TTTGCTGCCA ACATCGATTT GATGATGATT GA:G:ATATC CATATGTTGT CATTCTCTAA ACAAAAAGAC AATTTCTTAT A KJ542096 TTACGGTTAT TTTGCTGCCA ACATCGATTT GATGATGATT GA-G:ATATC CATATGTTGT CATTCTCTAA ACAAAAAGAC AATTTCTTAT A KJ542089 TTACGGTTAT TTTGCTGCCA ACATCGATTT GATGATGATT GA:G:ATATC CATATGTTGT CATTCTCTAA ACAAAAAGAC AATTTCTTAT A

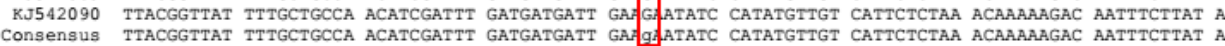

Figure 1. Alignment of Der $\mathrm{p} 1$ allergen group 1 amino acid (Der $\mathrm{p} 1$ proteins). The variation areas are indicated by a red block 
Table 1. Geographical polymorphisms of the Der $\mathrm{p} 1$ genes

\begin{tabular}{|c|c|c|c|c|c|c|c|c|c|}
\hline \multirow{2}{*}{$\begin{array}{c}\text { Accession } \\
\text { number }\end{array}$} & \multirow{2}{*}{$\begin{array}{l}\text { Geographical } \\
\text { position }\end{array}$} & \multicolumn{8}{|c|}{ DNA sequence position } \\
\hline & & 190 & 463 & 640 & 645 & 751 & 787 & 827 & 1084 \\
\hline AA1 & Minahasa Indonesia & $\mathrm{G}$ & $\mathrm{C}$ & $\mathrm{T}$ & A & $\mathrm{C}$ & $\mathrm{C}$ & $\mathrm{T}$ & $\mathrm{C}$ \\
\hline KJ542093 (1) & Lenoir US & $\mathrm{G}$ & $\mathrm{C}$ & $\mathrm{T}$ & A & $\mathrm{C}$ & $\mathrm{C}$ & $\mathrm{T}$ & $\mathrm{C}$ \\
\hline KJ542087 (2) & Rawalpindi Pakistan & G & $\mathrm{T}$ & $\mathrm{C}$ & A & $\mathrm{C}$ & G & A & G \\
\hline KJ542086 (3) & Lenoir US & G & $\mathrm{T}$ & $\mathrm{C}$ & $\mathrm{R}$ & $\mathrm{C}$ & G & A & G \\
\hline KJ542090 (4) & Rawalpindi Pakistan & G & $\mathrm{T}$ & $\mathrm{C}$ & A & $\mathrm{Y}$ & G & A & G \\
\hline KJ542089 (5) & Rawalpindi Pakistan & G & $\mathrm{T}$ & $\mathrm{C}$ & A & $\mathrm{T}$ & G & A & G \\
\hline KJ542096 (6) & Rawalpindi Pakistan & $\mathrm{R}$ & $\mathrm{T}$ & $\mathrm{C}$ & $\mathrm{R}$ & $\mathrm{C}$ & $\mathrm{G}$ & W & $\mathrm{G}$ \\
\hline Consensus & & $\mathrm{g}$ & $\mathrm{t}$ & $\mathrm{c}$ & $\mathrm{a}$ & c & $\mathrm{g}$ & . & $\mathrm{g}$ \\
\hline
\end{tabular}

Note: R: purine (A or $\mathrm{G})$; $\mathrm{Y}$ : pirimidin $(\mathrm{C}$ or $\mathrm{T})$; $\mathrm{W}$ : A or T: Weak base pair

A study by Piboonpocanun et al. (2006) has revealed an enlarged data set that suggest preferred amino acid substitutions in residues 19,81 , and 215 of Der $p 1$ as well as the irregular changes. Meanwhile, Der $p 2$ has had frequent variations with clusters of amino acid substitutions, although, amongst the 17 sequences, the Der p 2.0101 is absent. Comprehensive sequence information on variants is crucial to optimize allergen formulations and also beneficial for genetic engineering and structurefunction analyses of many important allergens (Thomas 2010). The presence of epitope residues in areas of high allergen homology may indicate the cross-reactivity Der $p$ 1 allergens, while the presence of IgE-binding epitope in the variable region may lead to species specificity (Chruszcz et al. 2012). As cross-reactivity and specificity of monoclonal antibodies can be highly affected by mutations in several IgE-binding epitopes, the allergen extracts development should encourage allergen diversity inter or intraspecies be considered in the process for more proper result. This emphasizes that accurate and appropriate identification and characterization of representative variants at specific geographic locations are essential and necessary (Karki et al. 2015). Regional amino acid sequence polymorphisms and the extent to which these polymorphisms are poorly studied and understood. The data of such information are limited for most countries, including the US and Pakistan. The study of internal polymorphisms in the group 1 allergen gene DP was the important species of house dust mite medical methods, collected in the US and Pakistan (Shafique et al. 2014).

\section{Der p 1 allergen protein polymorphisms}

The deduced amino acid sequences show the existence of 5 silent and 3 non-synonymous mutations as follows: Histidine H146 to Tyrosine Y146, Alanine A220 to Valine V220, and any amino acid X220, and Tyrosine T232 to Serine S232 (Table 2). The alignment results of the amino acid sequences of Der p 1 allergen can be seen in Figure 2. The variation areas marked by red block, meanwhile the active site areas are indicated by blue block.

Table 2. Amino acid polymorphisms in Der p 1 allergen

\begin{tabular}{lccc}
\hline \multirow{2}{*}{ Accession number } & \multicolumn{3}{c}{ Amino Acid Position } \\
\cline { 2 - 4 } & $\mathbf{1 4 6}$ & $\mathbf{2 2 0}$ & $\mathbf{2 3 2}$ \\
\hline AA1 & $\mathrm{H}$ & $\mathrm{A}$ & $\mathrm{T}$ \\
AHZ89364 (1) & $\mathrm{H}$ & $\mathrm{A}$ & $\mathrm{T}$ \\
AHZ89358 (2) & $\mathrm{Y}$ & $\mathrm{A}$ & $\mathrm{S}$ \\
AHZ89357 (3) & $\mathrm{Y}$ & $\mathrm{A}$ & $\mathrm{S}$ \\
AHZ89361 (4) & $\mathrm{Y}$ & $\mathrm{A}$ & $\mathrm{S}$ \\
AHZ89360 (5) & $\mathrm{Y}$ & $\mathrm{V}$ & $\mathrm{S}$ \\
AHZ89367 (6) & $\mathrm{Y}$ & $\mathrm{X}$ & $\mathrm{S}$ \\
Consensus & $\mathrm{y}$ & $\mathrm{a}$ & $\mathrm{S}$ \\
\hline
\end{tabular}

Note: H: Histidine (polar); Y: Tyrosine (polar); A: Alanine (nonpolar); V: Valine (non-polar); X: any amino acid; T: Threonine (polar); S: Serine (polar)

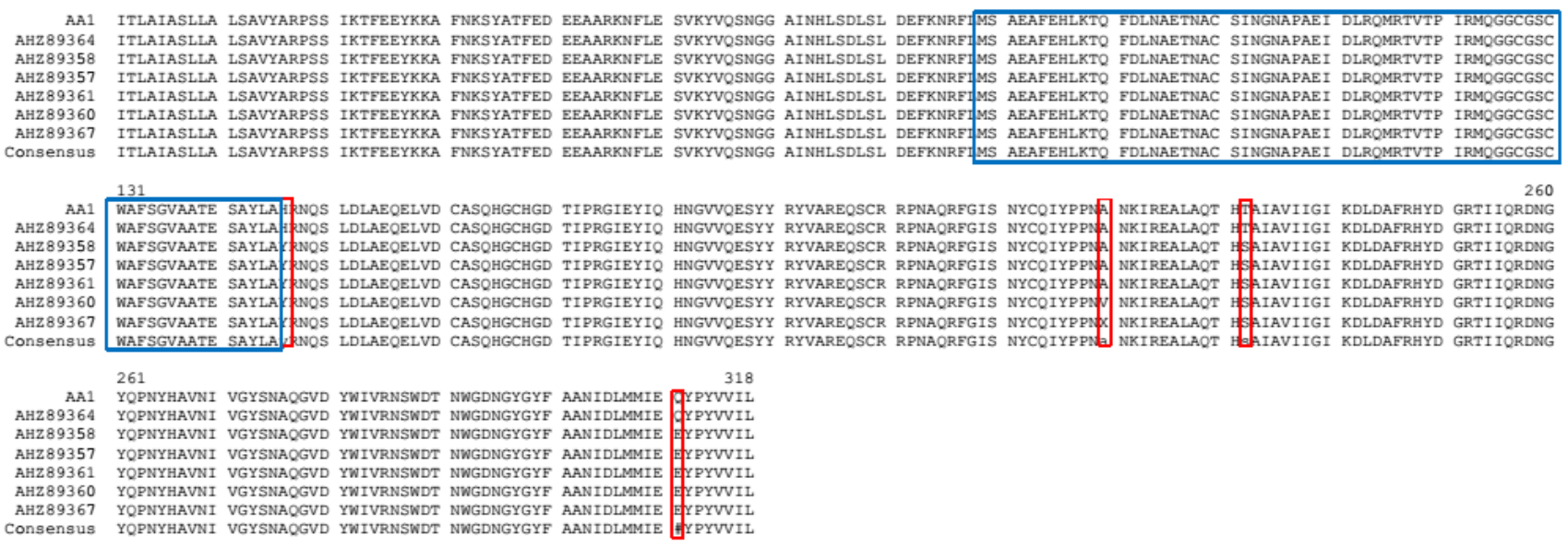

Figure 2. Analysis of amino acid alignment of Der $\mathrm{p} 1$ allergen. The variation areas are indicated by a red block and the active site areas are indicated by a blue block 
Polymorphism in these amino acids is a polarity change from polar to polar and non-polar to non-polar. These dynamics do not change the structure or function of the allergen protein. This result is compatible with several other studies, suggesting that very few polymorphisms occur in the allergen gene, in which all but one mutation are silent and do not affect the protein primary structure where substantial amino acid variation is located in DP, however, the variations are less compared with the previously reported (Piboonpocanun et al. 2006).

Since Der p 1 was first discovered to be a cysteine protease (proteolytic enzyme), there have been many allergens with proteolytic activity explored. The protease groups in DP are more frequently transcribed compared to the average of this species (Gough et al. 2014). Cysteine protease, such as Der p 1, dominates the distribution of other protease types in allergen. In general, cysteine protease serves about half of the protease transcription, and Der p 1 in particular responsible for $22 \%$ of the total protease transcript (López-Rodríguez 2019). In the protease stability analysis, the allergen groups (Der $\mathrm{p} \mathrm{1,}$ Der p 3, Der p 6, and Der p 9) were proven to be more stable than average. Statistically, as well, the protease allergen was simultaneously more expressed and stable than the DP group (Shafique et al. 2014).

Amino acid polymorphisms can affect the reactivity of the IgE bonds from allergens (Liao et al. 2015). A single amino acid mutation may alter the production of inflammatory cytokines specific $\mathrm{T}$ cells for Der $\mathrm{p} 1$ (Chevigne et al. 2018). It is thought that these mutations can affect the innate allergenicity of certain variants and encourage the frequency of $\operatorname{IgE}$ binding by increasing epitope diversity (Jeong et al. 2012). There are more than half of mite allergic patients percentage that react positively to the Der $\mathrm{p} 1$ protein, thus only Der $\mathrm{p} 1$ is thought to be the primary allergen. This fact is proven to be more accurate with the data gathered from the latest studies, suggesting that the prevalence of Der $\mathrm{p} 1$ reactivity is around 75\% (Chevigne et al. 2018). In a recent analysis of $E$. coli gene obtained from DP feces, polymorphic residues have been found in regions containing the predetermined T-epitope. The data containing polymorphism information reported in this study will help to understand both the immune response of mite allergens and the development of peptide-based immunotherapy reagents specifically for mite-related allergies. However, as allergens can also be found clearly in intact mite extracts used in the diagnosis of mite allergy and immunotherapy, characterization will be worth it for medical purposes.

\section{Phylogenetic analysis of Der $p 1$ genes and Der $p 1$ protein of DP from several locations}

Figure 3 shows the phylogenetic analysis of Der $p 1$ genes from Indonesia, United States (US), and Pakistan. Based on Der p 1 gene, DP isolates AA1 from Indonesia is closely related to accession number KJ542093 from Lenoir US. In Figure 4, the position of the AA1 0 protein is highlighted in blue in the phylogenetic tree, in which the protein is identical to $\mathrm{P} 08176$ with a similarity value of $98.43 \%$

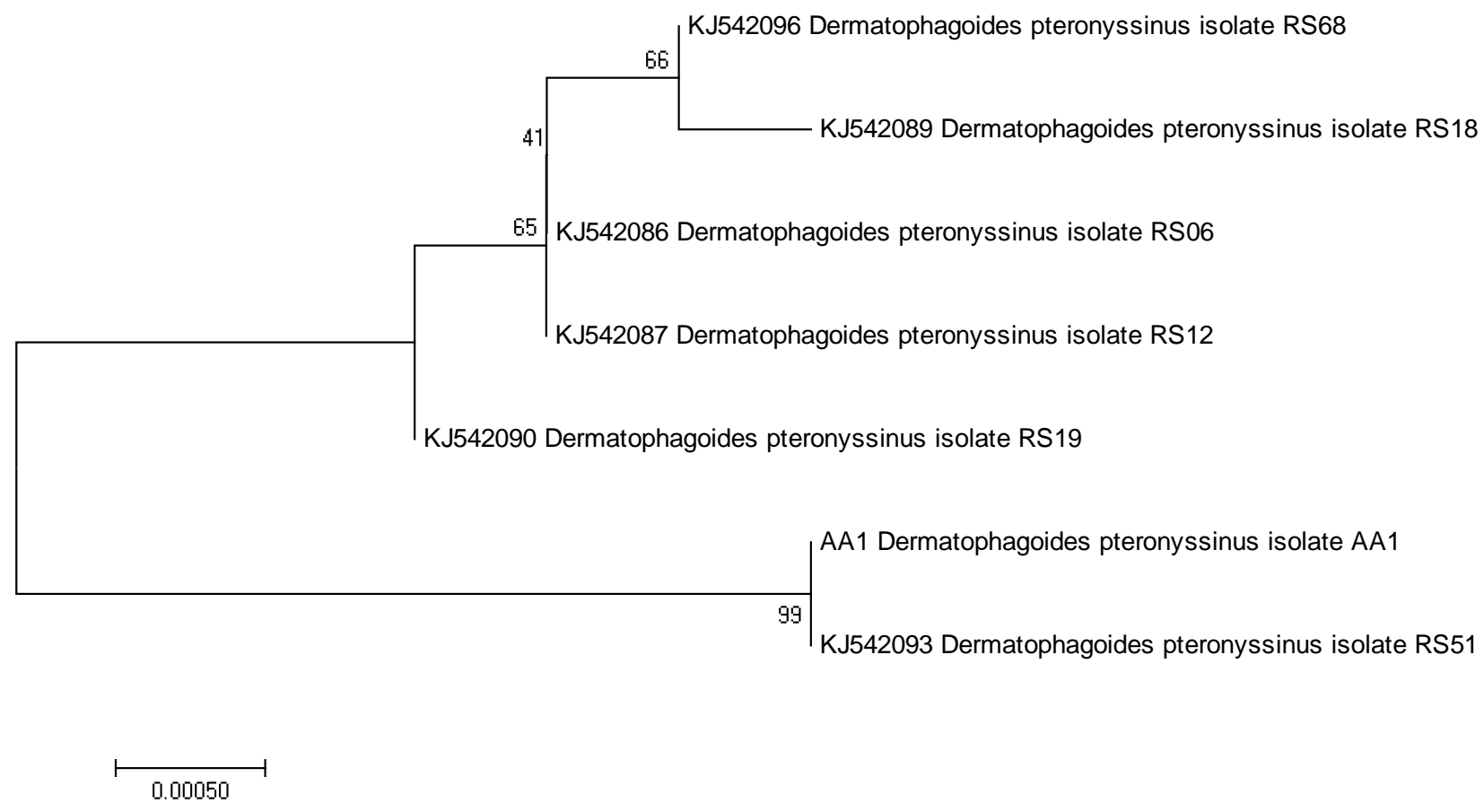

Figure 3. Taxonomic evolutionary relationships of the phylogenetic DP mite based on Der $\mathrm{p} 1$ gene by Neighbor-Joining method 


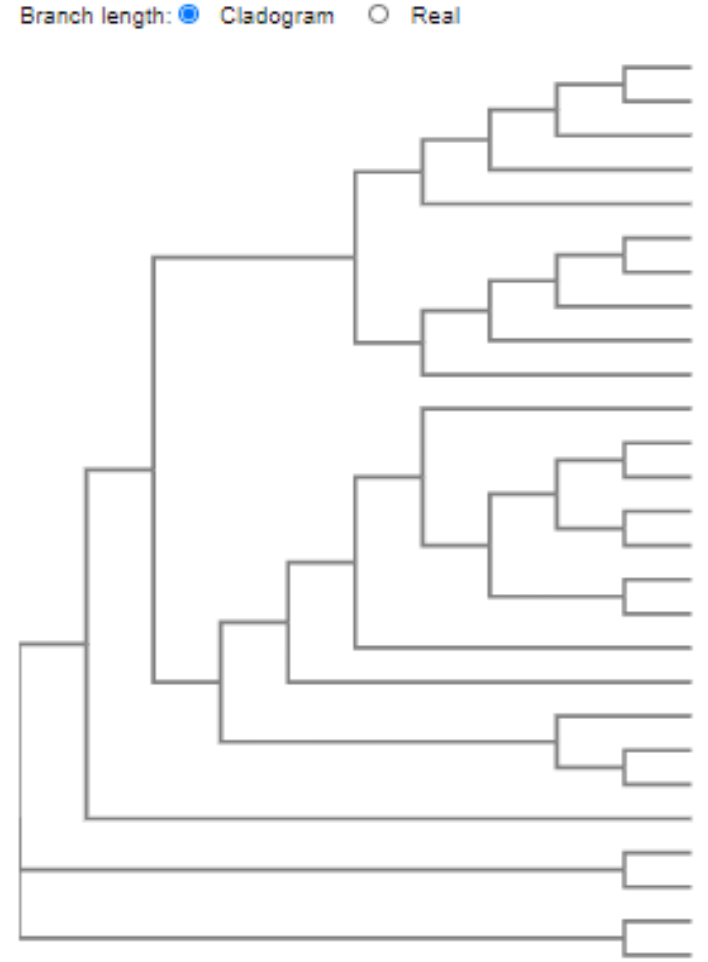

Figure 4. Phylogenetic tree of protein Der $\mathrm{p} 1$ by Clustal Omega

The phylogenetic tree (Figure 4) shows that Der $\mathrm{p} 1$ protein of DP (AA1 0) is highly identical to the partial preproenzyme Cysteine proteinase-1 DP AHZ89364.1 0. In the phylogenetic tree, they appear in one clade. Contrary to the research of evolutionary relationship among HDM, homology analysis of amino acid sequences of group 1 and 2 allergens, namely DP and E. Maynei, shows that Der $\mathrm{p} 1$ and Der $\mathrm{p} 2$ of DP have more resemblances to Eur $\mathrm{m} 1$ and Eur $\mathrm{m} 2$, compared to Der f 1 and Der f 2 from $D$. farinae. The phylogenetic tree shows DP parallel to E. maynei, but not to $D$. farinae, although DP and $D$. farinae are under the same genus according to morphological taxonomy. So it can be assumed that DP is more closely related to $E$. maynei than $D$. farinae at the evolutionary stage (Cui et al. 2010).

This study concludes that the Der $\mathrm{p} 1$ gene's polymorphisms and the amino acids of the Der $\mathrm{p} 1$ protein do not affect the structure and function of allergen proteins. Der p1 gene polymorphisms with five silent mutations and 3 non-synonymous substitutions do not change allergen proteins' structure or function to be used for vaccine design strategies in future perspectives.

\section{ACKNOWLEDGEMENTS}

The authors would like to thank the Entomology Program of Graduate Program, and Department of Biology, Faculty of Mathematics, and Natural Sciences, Sam Ratulangi University, Manado, North Sulawesi, Indonesia, as well as Department of Dermatovenereology, RD Kandou

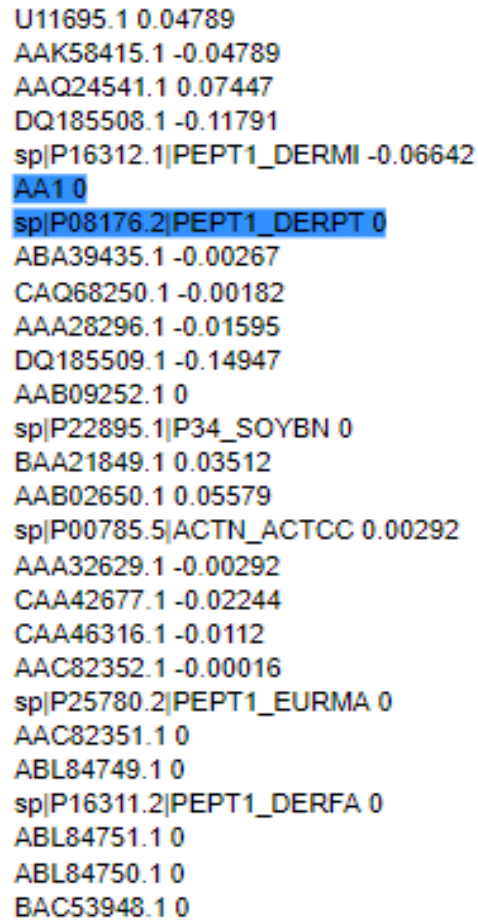

Hospital, Manado, North Sulawesi, Indonesia for providing the place to conduct the research.

\section{REFERENCES}

Altshul SF, Gish W, Miller W, Myers EW, Lipman DJ. 1990. BLAST (Basic Local Alignment Search Tool). J Mol Biol 215: 403-10. https://blast.ncbi.nlm.nih.gov/Blast.cgi.

Cao R, Shi Y, Chen S, Ma Y, Chen J, Yang J, Chen G, Shi T. 2017. dbSAP: single amino-acid polymorphism database for protein variation detection. Nucleic Acids Res 45: D827-D832. DOI: 10.1093/nar/gkw1096

Chevigne A, Jacquet A. 2018. Emerging roles of the protease allergen Der p 1 in house dust mite-induced airway inflammation. J Allergy Clin Immunol 142: 398-400.

Chruszcz M, Pomés A, Glesner J, Vailes LD, Osinski T. 2012. Molecular determinants for antibody binding on group 1 house dust mite allergens. J Biol Chem 287: 7388-7398.

Clancy S. 2008. Genetic mutation. Nat Educat 1 (1): 187

Corpet F. 1988. Multalin (Multiple Sequence Alignment with Hierarchical Clustering). Nucl Acids Res 16 (22): 10881-890.

Cui Y, Gao C, Zhou Y, Zhou P, Peng M, Lin Y, Peng J. 2010. Phylogenetic analysis of house dust mites. Cent Eur J Med 5 (1): 6974.

Cui, Y, Wang Q, Jia H. 2018. Consideration of methods for identifying mite allergens. Clin Transl Allergy 8:14. DOI: 10.1186/s13601-0180200-4

Herman J. 2014. Der p 1 is the primary activator of Der p 3, Der p 6 and Der $\mathrm{p} 9$ the proteolytic allergens produced by the house dust mite Dermatophagoides pteronyssinus. Biochim Biophys Acta 1840: 11171124.

Hizawa N, Freidhoff LR, Chiu YF, Ehrlich E. 1998. Genetic regulation of Dermatophagoides pteronyssinus-specific IgE responsiveness: A genome-wide multipoint linkage analysis in families recruited through 2 asthmatic sibs. J Allergy Clin Immunol 102 (3): 436-42. 
International Human Genome Sequencing Consortium. 2001. Initial sequencing and analysis of the human genome. Nature 409:860-921.

Izquierdo-Alvarez A. 2012. Differential redox proteomics allows identification of proteins reversibly oxidized at cysteine residues in endothelial cells in response to acute hypoxia. J Proteom75: 5449. 5462.

Jeong KY, Lee IY, Yong TS, Lee JH, Kim EJ. 2012. Sequence polymorphisms of Der f 1, Der p 1, Der f 2 and Der p 2 from Korean house dust mite isolates. Exp Appl Acarol 58: 35-42.

Jia X, Han B, Onengut-Gumuscu S, Chen W-M, Concannon PJ, Rich SS Raychaudhuri S, de Bakker PIW. 2013. Imputing Amino Acid Polymorphisms in Human Leukocyte Antigens. PLoS ONE 8(6): e64683,. DOI: 10.1371/journal.pone.0064683

Karki R, Pandya D, Elston RC, Ferlini C. 2015. Defining "mutation" and "polymorphism" in the era of personal genomics. BMC Med Genomics 8: 37. DOI: 10.1186/s12920-015-0115-z

Liao EC, Hsieh CW, Chang CY, Yu SJ, Sheu ML, Wu SM, Tsai JJ. 2015. Enhanced allergic inflammation of Der p 2 affected by polymorphisms of $M D-2$ Promoter. Allergy Asthma Immunol Res 7 (5): 497-506, DOI: 10.4168/aair.2015.7.5.497.

López-Rodríguez JC, Manosalva J, Cabrera-García JD, Escribese M, Villalba MM, Barber D, Martínez-Ruiz A, Batanero E. 2019. Human glutathione-S-transferase pi potentiates the cysteine-protease activity of the Der $\mathrm{p} 1$ allergen from house dust mite through a cysteine redox mechanism. Redox Biol 26: 101256. DOI 10.1016/j.redox.2019.101256.

Lusiastuti AM, Seeger H, Sugiani D, Mufidah T, Novita H. 2015. Deteksi polymorphisme dengan substitusi nukleotida tunggal pada Streptococcus agalactiae isolat lokal Indonesia. Media Akuakultur 10 (2): 91-95. [Indonesian]

Mix, Go E. coli Transformation Kit. Zymo Research Corp. www.zymoresearch.com.

Piboonpocanun S, Jirapongsananuruk O, Thomas WR, Malainual N, Vichnayond P. 2006. Genetic polymorphism of major house dust mite allergens. Clinical \& Experimental Allergy 36 (4):510-6. DOI: 10.1111/j.1365-2222.2006.02464.x

Resende RO, Ynoue LH, Miranda JS, de Almeida KC, de Oliveira Silva DA, Sopelete MC, Alves R, Gennari-Cardoso ML, Taketomi EA. 2019. IgE, IgG1, and IgG4 Reactivity to Dermatophagoides pteronyssinus Glycosylated Extract in Allergic Patients. Biomed Res Int Article ID 9840890. DOI: 10.1155/2019/9840890

Saitou M. and Mei M. 1987. The neighbor-joining method: A new method for constructing phylogenetic trees. Mol Biol Evol 4: 406 - 425.

Shafique RH, Klimov PB, Inam M, Chaudhary FR, O'Connor BM. 2014. Group 1 Allergen Genes in Two Species of House Dust Mites, Dermatophagoides farinae and D. pteronyssinus (Acari: Pyroglypidae): Direct Sequencing, Characterization and Polymorphism. PLoS One 9 (12): 114636. DOI:0.1371/journal.pone.0114636.

Tallei TE, Kolondam BJ. 2015. DNA barcoding of Sangihe nutmeg (Myristica fragrans) using matK gene. Hayati J Biosciences 22 (1): 41-47. DOI:10.4308/hjb.22.1.41

Thomas WR. 2010. Geography of house dust mite allergens. Asian Pac J Allergy Immunol 28: 211-224.

Toyobo Kod FX Neo (KFX-201). Target clone/Target clone plus. www.toyobo.co.jp/e/bio.

Toyobo. Target clone/Target clone plus. www.toyobo.co.jp/e/bio.

Twyman R. 2003. Mutation or polymorphism? Wellcome Trust website. http://genome.wellcome. ac.uk/doc_WTD020780.html

Verma S, Dixit R, Pandey KC. 2016. Der p 1 proteins: modes of activation and future prospects as pharmacological targets. Front Pharmacol 7:107.

Waldron R, Mc Gowan J, Gordon N, Mc Carthy C, Mitchell EB, Fitzpatrick DA. 2019. Proteonome and allergenome of the European house dust mite Dermatophagoides pteronyssinus. PLoS ONE 14(5):e0216171. DOI: 10.1371/ journal.pone.0216171 